\title{
Educação médica em saúde: relato de experiência em simpósio sobre mulher, planejamento familiar e direitos reprodutivos, no contexto da COVID-19
}

Medical education in health: experience report in a symposium about women, family planning and reproductive rights, in the context of COVID-19

Educación médica en salud: informe de experiencia en un simposio sobre mujeres, planificación familiar y derechos reproductivos, en el contexto del COVID-19 Centro Universitário São Lucas, Brasil

E-mail: pedrohenriquecborges@gmail.com Ítalo Cosmo Suarez de Sousa

ORCID: https://orcid.org/0000-0001-5309-028X Centro Universitário São Lucas, Brasil

E-mail: italosousa8@outlook.com

Victoria Mendes da Rocha Cardoso

ORCID: https://orcid.org/0000-0002-5239-2304 Centro Universitário São Lucas, Brasil E-mail: vicctoriamenddes@gmail.com

Flávio Aparecido Terassini

ORCID: https://orcid.org/0000-0001-9871-3989 Centro Universitário São Lucas, Brasil

E-mail: flavio@saolucas.edu.br

\section{Resumo}

Durante o isolamento social decorrente da pandemia da COVID-19, tem-se como um dos desafios continuar, de um modo inovador, a preparar os adolescentes para a vida sexual de forma segura, chamando-os à responsabilidade de cuidar de seu próprio corpo para que não ocorram situações futuras indesejadas como a contração de doenças sexualmente transmissíveis (ISTs) ou uma gravidez não planejada. Nesse sentido, via-se a necessidade de criação de um espaço de caráter tecnológico para a interação entre docentes especialistas para com os discentes do Centro Universitário São Lucas (UniSL), em Porto Velho/Rondônia, e a comunidade local no intuito de orientar quanto ao aconselhamento dos métodos contraceptivos definitivos, direitos sexuais, reprodutivos e liberdade da mulher além de uma sessão interativa sobre mitos e verdades quanto aos métodos contraceptivos.

Palavras-chave: Métodos contraceptivos; Direitos sexuais; Saúde da mulher; Planejamento familiar; Políticas públicas de saúde; COVID-19.

\footnotetext{
Abstract

During the social isolation resulting from the COVID-19 pandemic, one of the challenges is to continue, in an innovative way, to prepare teenagers for sexual life in a safe way, calling them to the responsibility of taking care of their own bodies to prevent unwanted situations such as contracting sexually transmitted diseases (STIs) or an unplanned pregnancy do not occur. In this sense, there was a need to create a technological space for interaction
} 
between expert professors and students at the São Lucas University Center (UniSL), in Porto Velho/Rondônia, and the local community in order to guide the counseling on definitive contraceptive methods, sexual rights, reproductive rights and women's freedom, also an interactive session on myths and truths about contraceptive methods.

Keywords: Contraceptive methods; Sexual rights; Women's health; Family planning; Public health policies; COVID19.

\section{Resumen}

Durante el aislamiento social derivado de la pandemia COVID-19, uno de los desafíos es continuar, de manera innovadora, preparando a las adolescentes para la vida sexual de manera segura, llamándolas a la responsabilidad de cuidar su propio cuerpo para que no se producen situaciones futuras no deseadas, como contraer enfermedades de transmisión sexual (ITS) o un embarazo no planificado. En este sentido, era necesario crear un espacio tecnológico de interacción entre profesores expertos y estudiantes del Centro Universitario São Lucas (UniSL), en Porto Velho / Rondônia, y la comunidad local para orientar la asesoría sobre métodos anticonceptivos definitivos, derechos sexuales, reproductivos y de libertad de las mujeres, así como una sesión interactiva sobre mitos y verdades sobre los métodos anticonceptivos.

Palabras clave: Métodos anticonceptivos; Derechos sexuales; La salud de la mujer; Planificación familiar; Políticas de salud pública; COVID-19.

\section{Introdução}

Estudos recentes (Woodworth et al., 2020), como o relatório do Centers for Diseases Control and Prevention, indicam que mulheres grávidas com infecção ativa pelo vírus de agente etiológico SARS-CoV-2 apresentam risco elevado de gravidade de doença em comparação com mulheres não grávidas, em idade reprodutiva. Nesse sentido, índices epidemiológicos demonstraram que tiveram maior taxa de internação em unidade de terapia intensiva (UTI), maiores índices de ventilação invasiva e um número maior de óbitos em comparação às mulheres não gestantes, também em idade reprodutiva. Importante salientar que, no Brasil, o Ministério da Saúde inseriu as gestantes e puérperas na classificação de alto grupo de risco, na pandemia, devido à imunidade e à elevada suscetibilidade à hipóxia (Avila \& Carvalho, 2020; Mascarenhas et al., 2020). Nesse sentido, vê-se a necessidade de promoção em saúde, inclusive no período de isolamento social decorrente da pandemia do COVID-19, no intuito de apontar os direitos reprodutivos conquistados pelas mulheres, discutir e abordar sobre planejamento familiar concomitantemente com prevenção e uso racional dos métodos contraceptivos adequados, evitando uma gravidez inesperada, na adolescência principalmente, durante uma pandemia (Ferreira et al., 2021).

Dados do Sistema Único de Saúde (SUS) demonstram que anualmente na década passada, se por um lado, cerca de 1,47 milhão de gestações foram planejadas, por outro lado 1,79 milhão não foram planejadas, ao que tudo indica, isso é reflexo da carência de políticas públicas efetivas na educação em saúde sexual na corroboração da construção da instituição família. É fundamental para os direitos sexuais das mulheres e/ou próprio casal propiciar esses conhecimentos científicos sobre planejamento familiar a fim de garantir uma liberdade e autonomia para uma organização familiar que, aglutina-se os valores tanto sociais pessoais quanto os valores econômicos de renda de cada família. Anualmente 122.523 partos prematuros são atribuídos a gestações não planejadas, dados que consolidam a tese de que no intuito de minimizar danos na saúde pública, são necessárias políticas públicas e ações de promoção em saúde sexual no Brasil (Bahamondes et al., 2017).

O planejamento familiar consiste em um conjunto de ações com a intenção de educar a população através da assistência a homens e mulheres quanto a seus papéis na construção de uma família, atuando na prevenção primária da saúde, aconselhando sobre a reprodução, métodos contraceptivos, explanando a importância de planejar a gestação, porém até o presente permanece principalmente voltado para as mulheres com o desejo de gestar, levando a participação masculina ser escassa tanto na saúde reprodutiva tanto quanto na saúde sexual.

Nesse sentido, vê-se a necessidade de promoção em saúde, inclusive no período de isolamento social decorrente da pandemia do COVID-19, no intuito de apontar os direitos reprodutivos conquistados pelas mulheres, discutir e abordar sobre planejamento familiar concomitantemente com prevenção e uso racional dos métodos contraceptivos adequados, evitando uma 
gravidez inesperada, na adolescência principalmente, durante uma pandemia (Ferreira \& Souza, 2021).

\section{Metodologia}

Trata-se de um estudo do tipo relato de experiência com metodologia dedutiva com abordagem qualitativa que ocorreu no ambiente virtual. Segundo Pereira et al. (2018, p.28), a metodologia dedutiva:

Partindo das teorias e leis, na maioria das vezes prevê a ocorrência dos fenômenos particulares (conexão descendente); se o conhecimento é insuficiente para explicar um fenômeno, surge o problema; para expressar as dificuldades do problema são formuladas hipóteses; das hipóteses deduzem-se consequências a serem testadas ou falseadas (tornar falsas as consequências deduzidas das hipóteses).

Realizou-se uma extensa revisão bibliográfica acerca do tema elaborado com base em pesquisa de artigos científicos nas plataformas Scientific Electronic Library Online (SciELO), UptoDate, National Center for Biotechnology Information (NCBI), Google Acadêmico e PubMEd, nos idiomas inglês, português e espanhol. Dentre os critérios de inclusão para pesquisa bibliográfica, tem-se artigos publicados nos anos de 2015 até 2021, as palavras-chave utilizadas como descritores foram: planejamento familiar, direitos reprodutivos, saúde sexual, métodos contraceptivos, saúde da mulher, dentre os critérios de exclusão estão a incompatibilidade com o tema e artigos publicados antes de 2015 no intuito de ter ciência e delimitar a problemática da intervenção.

Optou-se por realizar uma intervenção de promoção em saúde preventiva de saúde da mulher, por meio de plataformas tecnológicas online através de uma conferência remota com o software da Zoom Video Communication® que combina por videoconferência o simpósio e a interação de dúvidas durante todo o momento do evento de extensão, concomitantemente, foi utilizado como catalisador de aprendizagem o software da Kahoot!@, essa plataforma foi ajustada para perguntas relacionadas às palestras, logo, um aprendizado baseado em jogos interativos auferindo proveito dessa tecnologia educacional para o engajamento participativo.

Para divulgação do evento, utilizamos canais como Instagram ${ }^{\circledR}$ com a colaboração de canais de comunicação voltados a saúde adjacentes ao tema, tais quais Associação de Obstetrícia e Ginecologia de Rondônia; Liga Acadêmica de Ginecologia e Obstetrícia de Rondônia; Liga Acadêmica de Saúde Coletiva de Rondônia; De Novo Não (Instagram médico educativo de saúde da mulher) e plataformas online de comunicação como o WhatsApp Messenger ${ }^{\circledR}$ e Telegram Messenger ${ }^{\circledR}$ compartilhando em grupos de pessoas da comunidade local, principalmente os de alunos de graduação em medicina.

\section{Resultados e Discussão}

O evento contemplou diversos aspectos no campo da contracepção sexual, inicialmente com a interlocução de um enfermeiro especialista na área e docente nos cursos de medicina da UniSL e na Universidade Federal de Rondônia. Ele abordou sobre o aconselhamento dos métodos contraceptivos definitivos incluindo, além dos procedimentos cirúrgicos laqueadura e vasectomia, e contraceptivos reversíveis de longa duração (LARC) como o dispositivo intrauterino (DIU) e os implantes subdérmico de etenogestrel, ponderando durante a apresentação a indicação mais adequada para cada mulher e suas especificidades e como um médico generalista deve seguir com a propedêutica clínica nas variadas situações.

Enfatizou-se que o DIU pode ser manejado pelo enfermeiro ou pelo médico responsável pelo atendimento enfermeiro, pois a inserção do DIU em ambulatório pelo enfermeiro se demonstrou tão segura quanto pelo médico (Trigueiro et al., 2021). Um estudo de 2020, demonstra que, palestras podem ter impacto significativo nas opiniões dos participantes sobre a eficácia dos métodos contraceptivos, fazendo com que eles tivessem escolhas mais informadas (Berardi et al., 2020). Trata-se de uma responsabilidade de uma equipe de saúde multidisciplinar em que as formas educacionais incluem o uso de estratégias e técnicas pedagógicas para socializar o conhecimento o que demanda um treinamento de profissionais de saúde nos cuidados 
com a vida (Ferreira et al., 2021).

Em relação ao implante subdérmico de etenogestrel foi abordado o quão grande é a sua eficiência na prevenção de um gravidez, sendo considerado o método mais seguro com 99,9\% de eficácia, melhor do que os métodos definitivos contracepção, atuando na disfunção ovariana (Rios et al., 2021). Esse contraceptivo possui o comprimento de $4 \mathrm{~cm}$, é feito de plástico e possui $68 \mathrm{mg}$ de etenogestrel com liberação lenta e com duração de três anos, sendo sua inserção realizada com anestesia local e é feita na face inferior do braço não dominante da paciente devendo o implante ser introduzido entre o primeiro e o quinto dia do ciclo menstrual, como todo método possui benefícios e efeitos adversos sendo eles: cefaleia $(15,3 \%)$, mastalgia (10,2\%), ganho de peso (11,8\%), acne (11,4\%), diminuição da libido (2,3\%) e labilidade emocional $(5,7 \%)$ seus efeitos adversos e os benefícios além da não concepção, inclui amenorreia (na maioria de suas usuárias), diminuição da dismenorreia e dos sintomas pré-menstruais (Braga \& Vieira, 2015).

O uso de plataformas online para abortar e discutir sobre o planejamento familiar, não se restringe apenas a palestras, simpósios, rodas de conversas, segundo Ferreira \& Souza et al. (2021), o emprego da telessaúde mostrou vantagens no atendimento de mulheres que buscavam aconselhamento sobre métodos de anticoncepção e como fazê-los e sanar dúvidas quando a questão é planejar uma gravidez de forma segura ou como preveni-la, o que se tornou mais confortável tanto para as paciente como para os profissionais os quais puderam continuar em isolamento atendendo e garantindo o conforto de suas pacientes.

O planejamento familiar consiste em programar o número de filhos que uma mulher ou casal deseja ter, quando serão gerados e quais as formas para ajudar neste caminho, utilizando métodos contraceptivos para alcançar esse objetivo no tempo estipulado, o que contribui para a liberdade de escolha das mulheres, interferindo positivamente nas reduções de gravidez na adolescência, riscos durante as gestações, além de ajudar a prevenir IST's, ou seja, sendo um suporte indispensável na saúde da mulher (RETS, 2016; Parmejiani et al., 2021). De acordo com o consenso de especialistas (Febrasgo, 2020) é de grande importância a aderência ao planejamento familiar principalmente neste período de pandemia, ressaltando que o uso de métodos contraceptivos deve ser mantido por aquelas que já fazem uso regular, e paralelamente, deve-se orientar que se evite interrupções, para as mulheres em idade reprodutiva que estão procurando por um método contraceptivo, deve-se orientá-las de forma correta quanto ao seu uso e também informar sobre os locais para a retirada, como por exemplo em Unidades Básicas de Saúde, com o objetivo de adiar gestações nesse período. Embora ainda não existem evidências robustas em relação à transmissão vertical, há uma certa apreensão com as possíveis intercorrências que possam ocorrer no decorrer da gestação (Febrasgo, 2020).

Em seguida, uma palestrante convidada, que atua como defensora pública abordou os direitos reprodutivos da mulher, como a Lei $\mathrm{N}^{\circ} 8.080$ (Ministério da Saúde, 2020) que institui o SUS e o regulamenta em todo território nacional, dispondo para compreensão das condições para promoção, proteção e recuperação da saúde, paralelamente, comentou sobre as competências das três esferas de governo e das áreas de atuação da assistência terapêutica integral (Oliveira, 2016). Por fim, abordou a Lei No 9263/1996 que trata sobre o planejamento familiar como parte integrante do conjunto de ações de atenção à mulher respeitando atividades como concepção e contracepção, pré-natal no quesito de direitos da paciente, assistência ao parto, controle de ISTs e outros direitos que todas as pacientes e médicos deveriam saber (Yamamoto, 2017; Rocha et al., 2020).

No final, foi convidada uma ginecologista e doutoranda que recorreu, além do Zoom®, à plataforma online Kahoot!® para uma dinâmica entre os alunos sobre mitos e verdades quanto aos contraceptivos, perguntas como se a pílula contraceptiva oral alterava o desenvolvimento do crescimento; se a contracepção de emergência era uma "bomba" hormonal; se a pílula anticoncepcional oral engorda; ou se o uso de pílula anticoncepcional por logo prazo poderia interferir na fertilidade da mulher, entre outras sobre o uso adequado da contracepção e todas essas perguntas foram escaladas, pelo software, em ranking 
de competitividade entre os ouvintes, gerando ambiente inovador de competitividade do conhecimento e interação ainda maior. Principalmente nessa última palestra, mostraram-se bem engajados os estudantes, respondendo perguntas e esclarecendo suas dúvidas, o que se tornou essencial para a consolidação da temática abordada.

Segundo Turok (2021) estudos indicam que os métodos contraceptivos de emergência como o acetato de uliprisal e levonogestrel (LNG) evitam a ovulação. O acetato de ulipristal pode ser usado até cinco dias depois de uma relação sexual desprotegida, é um método eficaz, contudo, sua taxa de gravidez é de 1,2\% a 1,8\% em um único ciclo. E o de LNG o risco de gravidez varia de $1,7 \%$ a 2,6\%, podem ser usados até cinco dias da relação. Esses contraceptivos de emergência devem ser usados por mulheres que se envolveram sexualmente com homens sem proteção adequada, no intuito de diminuir a chance de engravidar de forma indesejada. Os efeitos colaterais podem ser dor de cabeça, náuseas, dor abdominal, tonturas, fadigas e dismenorreia. São seguros quanto às esporádicas complicações trombogênicas dos anticoncepcionais hormonais. Esses métodos não são eficazes na prevenção das infecções sexualmente transmissíveis.

Para evitar ISTs, existem artifícios chamados contraceptivos de barreira, como o preservativo feminino e o preservativo masculino. O preservativo feminino é, basicamente, constituído por um anel externo que permanece em contato com a vulva, tem-se uma bainha que percorre a vagina e no interior, tem-se um outro anel interno ou uma esponja anteriormente ao colo do útero.

O preservativo feminino, além de evitar uma gravidez, protege contra secreções durante a relação sexual ao agir como barreira entre os órgãos genitais (Workowski et al., 2015) e uma das vantagens de seu uso é que não precisa de prescrição médica, além disso é disponibilizado gratuitamente pelo SUS pode estimular melhor o clitóris durante a relação sexual. Quanto à eficácia do preservativo feminino, tem-se que as taxas estimadas de gravidez inesperada durante o primeiro ano de uso em casais é de 5 até 21 por cento, logo, recomenda-se o uso concomitante de outro método contraceptivo além do de barreira para aumentar a eficácia. A conduta depende da orientação médica e da opção da paciente/casal (Hoke et al., 2021).

O preservativo masculino age como método de barreira impedindo uma gravidez e ISTs e, assim como o preservativo feminino, não é um método hormonal. Estudos recentes indicam uma eficácia de $98 \%$ quando usado adequadamente (WHO et al., 2018). Existe a possibilidade de fazer uso concomitante de outro método contraceptivo como os anticoncepcionais orais, vasectomia entre outros. As vantagens de seu uso é que protege contra ISTs (Workowski et al., 2015), é também distribuído gratuitamente pelo SUS, sendo ainda disponível para compra em diferentes cores, sabores e modelos, e alguns ainda possuem o efeito de retardar a ejaculação precoce masculina (Warner et al., 2021).

É relevante enfatizar, dentre os métodos contraceptivos apresentados, suas vantagens e desvantagens e principalmente a disponibilidade e oferta gratuita no SUS, no intuito de concomitantemente educar passivamente os estudantes e comunidade quanto a significância dessas políticas públicas em que estão inseridos socioeconomicamente (Santos et al., 2021).

A contabilização de inscritos online foi de 181 participantes, em sua maioria acadêmicos de medicina ou áreas voltadas à saúde da instituição UniSL, como também um volume considerável de especialistas na área como ouvintes que saudaram os palestrantes e elogiaram a iniciativa inovadora e contribuíram com perguntas e apontamentos importantes acerca dos temas abordados em cada palestra, além disso, teve-se também uma presença significativa de pessoas da própria comunidade como ouvintes.

\section{Considerações Finais}

A experiência desse evento com a associação de diferentes plataformas online atualizadas que são tendência educacional não somente nesses últimos anos, mas principalmente no momento de isolamento pela pandemia, foi uma oportunidade inovadora de divulgar todas essas informações de educação e promoção em saúde resultando em melhores cuidados médicos profiláticos para com o paciente e autocuidado (Ferreira et al., 2021). Importante apontar que o simpósio 
contou com um espectro de amplitude substancial de participantes graças à disponibilidade desses softwares online próprios para esse tipo de evento que não necessita necessariamente de uma localidade física para o evento e, até mesmo, por outros softwares auxiliando na sua divulgação pelas redes sociais.

Portanto, foi perceptível que a criação desse projeto inovador de caráter tecnológico possibilitou a integração do conhecimento de saúde da mulher e que a ampliação do contato através dessas diversas plataformas tecnológicas assegurou um melhor ensino em saúde de propedêutica para a formação de um médico generalista e, além disso, sanar medos, crenças equivocadas e preconceitos do uso adequado dos métodos contraceptivos para os acadêmicos e à comunidade. O feedback dos ouvintes foi expressivamente positivo o que confirmou a necessidade suprida da manutenção de conhecimentos relacionados à saúde feminina serem abordados com uma metodologia de afunilamento em um âmbito acadêmico de educacional de formação médica indo até ao alcance da população local com uma linguagem mais coloquial e de forma interativa fazendo com que os participantes ficassem mais focados ao decorrer do evento.

O desafio que permanece é o impacto positivo na saúde pública por meio da propagação de informações qualitativas quanto ao planejamento familiar e a continuidade desse projeto para um futuro evento regional e ou nacional. Importante salientar, por fim, os percalços de seguimento sem uma garantia de acesso à internet de qualidade para todos os discentes, pois é insuficiente e necessita da intervenção dos gestores para não haver prejuízo na aprendizagem daqueles menos favorecidos (Silva et al., 2021), ponderando-se aos cuidados com a saúde mental dos discentes e docentes das instituições (Ramos et al., 2021). Além disso, deve-se ficar vigilante em caso de processos de limitação de aprendizagem online dos estudantes e sempre procurar um mecanismo de transposição didática de abordagem humanística no meio acadêmico e com a comunidade (Ramos et al., 2021).

Por fim, para trabalhos de intervenção futuros, recomenda-se a publicação em canais de comunicação como Instagram ${ }^{\circledR}$, YouTube ${ }^{\circledR}$ e elaboração de vídeos lúdicos, curtos, com base em evidências científicas e artigos recentes, no intuído de promoção em saúde da mulher. Convidando, assim, o internauta do entretenimento ao conhecimento qualitativo e bem embasado sobre saúde da mulher.

\section{Referências}

Avila, W. S., \& Carvalho, R. C. (2020). COVID-19: Um Novo Desafio para a Cardiopatia na Gravidez. Sociedade Brasileira de Cardiologia, 115(1), 1-4. https://doi.org/10.36660/abc.20200511

Bahamondes, L., Fernandes, A., \& Monteiro, I. (2017). Barriers to Implementing and Consolidating a Family Planning Program that would meet Brazilian Needs. Revista Brasileira de Ginecologia, 39(08), 373-375. https://doi.org/10.1055/s-0037-1604423

Berardi, M. C., Mendes-Rodrigues, C., \& Paro, H. B. M. S. (2020). Contraceptive counseling lectures do not influence decision making in family planning services. Ciência e Saúde Coletiva, 25(6), 2369-2376. https://doi.org/10.1590/1413-81232020256.26472018

Braga, G. C., \& Vieira C. S. (2015). Anticoncepcionais reversíveis de longa duração: Implante Liberador de Etonogestrel (Implanon®). FEMINA, 43(1), 7-14. http://files.bvs.br/upload/S/0100-7254/2015/v43nsuppl1/a4849.pdf

Brasil. (2020). Ministério da saúde. Biblioteca Virtual em Saúde. Lei no 8080: 30 anos de criação do Sistema Único de Saúde (SUS). http://bvsms.saude.gov.br/ultimas-noticias/3295-lei-n-8080-30-anos-de-criacao-do-sistema-unico-de-saude-sus

Febrasgo (2020). Anticoncepção durante a pandemia por COVID-19. https://www.febrasgo.org.br/es/covid19/item/1002-anticoncepcao-durante-a-pandemiapor-covid-19r

Ferreira, A., L. C. G., \& Souza, A. I. (2021). O papel da telessaúde nos serviços de saúde sexual e reprodutiva em resposta à COVID-19. Revista Brasileira de Saúde Materno Infantil, 21(1), 323-326. https://doi.org/10.1590/1806-9304202100S100019

Ferreira, D. S., Ramos, F. R. S., \& Teixeira, E. (2021). Práxis educativa de enfermeiros da Estratégia Saúde da Família, 2021. Revista Brasileira de Enfermagem,74(2), e20200045. https://doi.org/10.1590/0034-7167-2020-0045

Hoke, T., Stone, K. M., Steiner, M. J., \& Warner, L. (2021). Female condoms. UpToDate. https://www.uptodate.com/contents/femalecondoms?search=preservativo\%20feminino\&source=search_result\&selectedTitle=1 144\&usage_type=default\&display_rank=1 
Mascarenhas, V. H. A., Caroci-Becker, A., Venâncio, K. C. M. P., \& Baraldi, N.G. (2020). Recomendações assistenciais à parturiente, puérpera e recémnascido durante a pandemia de COVID-19: revisão de escopo. Revista Latino-Americana de Enfermagem, 28, e3359. https://doi.org/10.1590/15188345.4596 .3359

Oliveira, J. (2016). Efetividade do direito à saúde: uma análise sob um contexto de crise financeira e constitucional. [Dissertação de Pós-graduação em Direito, Universidade do Oeste de Santa Catarina]. Biblioteca Virtual em Saúde. https://pesquisa.bvsalud.org/portal/resource/pt/sus-35601

Parmejiani, E. P., Queiroz, A. B. A., Pinheiro, A. S., Cordeiro, E. M., Moura, M. P. V., \& Paula, M. B. M. de. (2021). Saúde sexual e saúde reprodutiva da população ribeirinha: revisão integrativa. Revista da Escola de Enfermagem da USP, 55, e03664. https://doi.org/10.1590/S1980-220X2019033103664

Pereira, A. S., Shitsuka, D. M., Parreira, F. J., \& Shitsuka, R. (2018). Metodologia da pesquisa científica. https://repositorio.ufsm.br/bitstream/handle/1/15824/Lic_Computacao_Metodologia-Pesquisa-Cientifica.pdf?sequence=1

Ramos, S. C. S., Brochin, L. F., Carneiro, A. L. B., Júnior, O. C. R., Albarado, K. V. P., \& Martins, T. M. (2021). Ensino, monitoria e promoção da saúde em tempos de pandemia da COVID-19. Research, Society and Development, 10(8), e45410817544. http://dx.doi.org/10.33448/rsd-v10i8.17544

RETS - Rede Internacional de Educação de Técnicos em Saúde. (2016). Planejamento familiar/Contracepção. https://www.rets.epsjv.fiocruz.br/planejamentofamiliarcontracepcao

Rios, A. R., Sena, A. D., Krug, B. R., Dantas, E. K. de O., Ferronato, E. C. B., Bomfim, J. Q., Oliveira, L. A. de, Ferreira, P. C. C. M., Moura, V. G. de C., \& Guimarães, R. M. G. C. Fatores relacionados à escolha de métodos contraceptivos na adolescência: uma revisão de literatura. Revista Eletrônica Acervo Saúde, 13(5), e6942. https://doi.org/10.25248/reas.e6942.2021

Rocha, A. F., Gomes, K. R. O., \& Rodrigues, M. T. P. (2020). Impacto da intenção de engravidar sobre a amamentação na primeira hora pós-parto. Revista Ciência e Saúde Coletiva, 25(10), 4077-4086. https://doi.org/10.1590/1413-812320202510.00292019

Santos, L. F. S., Cassemiro, P. M. S., \& Souza, P. A. de. (2021). Conhecimento dos Acadêmicos do curso de graduação em Medicina sobre o Sistema Único de Saúde. Research, Society and Development, 10(8), e2310815863. http://dx.doi.org/10.33448/rsd-v10i8.15863

Silva, P. H. S., Faustino, L. R., Sobrinho, M. S. O., \& Silva, F. B. F. (2021). Educação remota na continuidade da formação médica em tempos de pandemia: viabilidade e percepções. Revista Brasileira de Educação Médica, 45(1), e044. https://doi.org/10.1590/1981-5271v45.1-20200459

Trigueiro, T. H., Lima, G. S., Borges, R., Guimarães, P. R B., Souza, R. R. K., \& Wall, M. L. (2021). Inserção de dispositivo intrauterino por médicos e enfermeiros em uma maternidade de risco habitual. Revista Gaúcha de Enfermagem, 42, e20200015. https://doi.org/10.1590/1983-1447.2021.20200015

Turok, D. (2021). Emergency contraception. UpToDate. https://www.uptodate.com/contents/emergency-contraception

Warner, L., Steiner, M. J., \& Stone, K. M. (2021). External (formerly male) condoms. UpToDate. https://www.uptodate.com/contents/external-formerly-malecondoms?search=Male\%20condoms\&source=search_result\&selectedTitle=1 150\&usage_type=default\&display_rank=1

Woodworth K. R., Olsen E. O., Neelam V., Lewis, E. L., Galang, R. R., Oduyebo, T., Aveni, K., Yazdy, M. M., Harvey, E., Longcore, N. D., Barton, J., Fussman, C., Siebman, S., Lush, M., Patrick P. H., Halai, U., Valencia-Prado, M., Orkis, L., Sowunmi, S., \& Tong, V. T. (2020). Birth and Infant Outcomes Following Laboratory-Confirmed SARS-CoV-2 Infection in Pregnancy - SET-NET, 16 Jurisdictions, Morbidity and Mortality Weekly Report, 69(44),16351640. http://dx.doi.org/10.15585/mmwr.mm6944e2

Workowski, K. A., Bolan, G. A., \& Centers for Disease Control and Prevention (2015). Sexually transmitted diseases treatment guidelines, 2015. Recommendations and reports, 64(3), 1-137. https://www.ncbi.nlm.nih.gov/pmc/articles/PMC5885289/

World Health Organization (WHO) \& Johns Hopkins. (2018). Family Planning: A Global Handbook for Providers. http://apps.who.int/iris/bitstream/handle/10665/260156/9780999203705-eng.pdf;jsessionid=3A12D5A4E37910C5590DE6C347F485B2?sequence=1

Yamamoto, S. T. (2017). Desencontros entre direitos e desejo da mulher e a decisão da equipe médica na prática da esterilização cirúrgica. [Tese de Doutorado, Universidade de São Paulo]. Biblioteca Digital USP. https://doi.org/10.11606/T.6.2018.tde-15022018-154445 